\title{
On interactions of static magnetic fields
}

\section{Ömer Zor*}

\begin{abstract}
We investigated the interaction energy of a Gilbertian magnetic charge with each of the "point" magnetic field sources. Finally we extrapolated a Dirac string can only be defined if there is at most one Dirac monopole in the medium. If there is only one Dirac monopole/string in the universe, the probability of detecting it is essential zero, such that Dirac's monopole would remain just a "theorist's particle".
\end{abstract}

K e yw ords: magnetic monopole, Dirac string, interaction energy

\section{Introduction}

Dirac $[1,2]$ constructed a quantization using the Hamiltonian for an electric charge interacting with the field of a fixed magnetic monopole, thus showing that the electric vector potential must be singular in the presence of a magnetic monopole. The singularity occurs on a line instantaneously extending outward from the monopole to spatial infinity (Dirac string). In the works of Dirac, Schwinger [3] and Zwanziger [4], it was shown that it is not possible to develop an electromagnetic theory of point electric and magnetic field sources without introducing the Dirac string or multi-valued potential [5].

The quantization of magnetic monopoles led to many works on the Hamiltonian for magnetic monopoles. One of the applications to systems containing both electric and magnetic charges is the interaction between two sources of magnetic fields. This problem has been investigated by several authors [6-12]. Comay introduced a paradox in his works [8-10], which is that the interaction of Gilbertian magnetic monopole with an Amperian magnetic dipole does not conserve energy. Lipkin and Peshkin [6,7] discussed similar paradoxes. They suggested that magnetic monopoles should be at the ends of strings of magnetic flux as described in Dirac's theory $[1,2]$ when calculating interaction energy. However, Lipkin and Peshkin could not provide a clear resolution of these paradoxes. Getino, Rojo and Rubio finally defined the interaction energy between an Amperian magnetic dipole and the Dirac string associated with a Gilbertian magnetic charge to be $-\vec{m}_{a} \cdot \vec{B}_{p}$, which resolves Comay's paradox to the extent that such strings are physical. However, McDonald calculated the field energy associated with a pair of Gilbertian magnetic charges with Dirac strings and produced a new paradox. McDonald [12] noted that one possible resolution of this paradox is that Gilbertian magnetic charges cannot exist.

\section{Formulation}

A Coulomb-like magnetic field at the position $\vec{r} \in R^{3}$, excited by a magnetic monopole $p$ fixed at $\vec{r}_{0} \in R^{3}$ where $\vec{r} \neq \vec{r}_{0}$, can be written in Gaussian units as

$$
\vec{B}_{p}(\vec{r})=\frac{p\left(\vec{r}-\vec{r}_{0}\right)}{\left|\vec{r}-\vec{r}_{0}\right|^{3}}
$$

The magnetic field of any given point-like charge distribution $4 \pi p \delta^{3}\left(\vec{r}-\vec{r}_{0}\right)$, where $\delta^{3}$ is the three-dimensional delta function, satisfies the following magnetic Gauss equation,

$$
\vec{\nabla} \cdot \vec{B}_{p}(\vec{r})=4 \pi p \delta^{3}\left(\vec{r}-\vec{r}_{0}\right) .
$$

Dirac [1] introduced the magnetic flux as $4 \pi p$, carried by the string that spans from the point charge $p$ to infinity. A string is infinitesimally thin solenoid, so we can define the magnetic field of the string using a delta function,

$$
\vec{B}_{s}=4 \pi p \vec{\delta}_{s}
$$

where the vector delta function $\vec{\delta}_{s}$ is parallel to $\hat{s}$ (unit vector tangent to the string), and $\vec{\delta}_{s}=\overrightarrow{0}$ for points not on the string.

For any two fields $\vec{F}_{1}, \vec{F}_{2}$ (both are electric or magnetic fields) in the volume $v$, which are excited by different electric or magnetic sources, the interaction energy between the two fields can be defined as

$$
U_{\text {int }}=\frac{1}{4 \pi} \int \vec{F}_{1} \cdot \vec{F}_{2} \mathrm{~d} v .
$$

In the following sections, we investigate the interaction energy of a Gilbertian magnetic charge with each of the "point" magnetic field sources.

\subsection{Two Gilbertian magnetic charges}

The magnetic field of Gilbertian magnetic charge $p$ at location $\vec{r}_{p}$ is $\vec{B}_{p}=-p \vec{r}_{p} / r_{p}^{3}$ with $\vec{\nabla} \cdot \vec{B}_{p}=4 \pi p \delta^{3}(\vec{r}-$

*Electrical-Electronics Engineering Department, Bursa Technical University, Bursa, TR-16310, Turkey, omer.zor@btu.edu.tr 
$\left.\vec{r}_{p}\right)$. The interaction energy of two Gilbertian magnetic charges can be written (in Gaussian units) as

$$
\begin{gathered}
U_{\mathrm{int}}=\frac{1}{4 \pi} \int \vec{B}_{p 1} \cdot \vec{B}_{p 2} \mathrm{~d} v=-\frac{1}{4 \pi} \int \vec{B}_{p 1} \cdot \vec{\nabla} \frac{p_{2}}{\left|\vec{r}-\vec{r}_{2}\right|} \mathrm{d} v \\
U_{\mathrm{int}}=-\frac{1}{4 \pi} \int\left(\vec{\nabla} \cdot \frac{p_{2} \vec{B}_{p 1}}{\left|\vec{r}-\vec{r}_{2}\right|}-\frac{p_{2} \vec{\nabla} \cdot \vec{B}_{p 1}}{\left|\vec{r}-\vec{r}_{2}\right|}\right) \mathrm{d} v .
\end{gathered}
$$

Using the divergence theorem, the integral can be transformed to

$$
U_{\text {int }}=-\frac{1}{4 \pi}\left(\int_{A \text { at } \infty} \frac{p_{2} \vec{B}_{p 1} \cdot \mathrm{d} \vec{A}}{\left|\vec{r}-\vec{r}_{2}\right|}-\int \frac{p_{2} \vec{\nabla} \cdot \vec{B}_{p 1}}{\left|\vec{r}-\vec{r}_{2}\right|} \mathrm{d} v\right) .
$$

$\vec{B}_{p 1} \propto 1 / r^{2}, \mathrm{~d} \vec{A} \propto r^{2}$, and therefore the first integral vanishes with $1 / r$ as $r$ becomes very large. And if we use the definition $\vec{\nabla} \cdot \vec{B}_{p 1}=4 \pi p_{1} \delta^{3}\left(\vec{r}-\vec{r}_{1}\right)$, we obtain

$$
U_{\text {int }}=\frac{p_{1} p_{2}}{r_{12}} \text {. }
$$

The implication is that the interaction of a Gilbertian magnetic charge with a Gilbertian magnetic charge does conserve energy.

\subsection{Two Gilbertian magnetic charges, each with an associated Dirac string}

The magnetic field of Gilbertian magnetic charge $p$ at location $\vec{r}_{p}$ with an associated Dirac string can be defined as $\vec{B}=\vec{B}_{p}+\vec{B}_{s}$. The interaction energy of two Gilbertian magnetic charges, each with an associated Dirac string, would be

$$
\begin{aligned}
& U_{\mathrm{int}}= \frac{1}{4 \pi} \int\left(\vec{B}_{p 1}+\vec{B}_{s 1}\right) \cdot\left(\vec{B}_{p 2}+\vec{B}_{s 2}\right) \mathrm{d} v \\
& U_{\mathrm{int}}=\frac{1}{4 \pi}\left(\int \vec{B}_{p 1} \cdot \vec{B}_{p 2} \mathrm{~d} v+\int \vec{B}_{p 1} \cdot \vec{B}_{s 2} \mathrm{~d} v\right. \\
&\left.+\int \vec{B}_{s 1} \cdot \vec{B}_{p 2} \mathrm{~d} v+\int \vec{B}_{s 1} \cdot \vec{B}_{s 2} \mathrm{~d} v\right) .
\end{aligned}
$$

Using the formulation in section 2.1 for first integral and assuming that the Dirac strings do not intersect, the fourth integral goes to zero. For the other integrals, we use the formulation,

$$
U=\frac{1}{4 \pi} \int \vec{B}_{p 1} \cdot \vec{B}_{s 2} \mathrm{~d} v=p_{2} \int \vec{B}_{p 1} \cdot \vec{\delta}_{s 2} \mathrm{~d} v .
$$

We can express the volume integral as an integral along the Dirac string times integrals over surfaces (A) penetrated by the string, then since $\vec{\delta}_{s}$ and $\mathrm{d} \vec{s}$ are parallel, they can be exchanged in the integrand,

$$
\begin{aligned}
U & =p_{2} \int_{s} \int_{\hat{n}_{A} \| \hat{s}}\left(\vec{B}_{p 1} \cdot \vec{\delta}_{s 2}\right)(\mathrm{d} \vec{s} \cdot \mathrm{d} \vec{A}) \\
& =p_{2} \int_{s} \int_{\hat{n}_{A} \| \hat{s}}\left(\vec{B}_{p 1} \cdot \mathrm{d} \vec{s}\right)\left(\vec{\delta}_{s 2} \cdot \mathrm{d} \vec{A}\right) \\
& =p_{2} \int_{s}\left(\vec{B}_{p 1} \cdot \mathrm{d} \vec{s}\right)=-p_{2} \int_{s} \vec{\nabla}\left(\frac{p_{1}}{\left|\vec{r}-\vec{r}_{1}\right|}\right) \cdot \mathrm{d} \vec{s} \\
& =\frac{p_{1} p_{2}}{\left|\vec{r}_{1}-\vec{r}_{2}\right|}=\frac{p_{1} p_{2}}{r_{12}},
\end{aligned}
$$

and we obtain

$$
U_{\mathrm{int}}=3 \frac{p_{1} p_{2}}{r_{12}}
$$

As a result of these calculations, the interaction of two Gilbertian magnetic charges, each with an associated Dirac string, does not conserve energy.

\subsection{Gilbertian magnetic charge and Gilbertian magnetic dipole}

The "point" magnetic dipole $\vec{m}_{G}$, consisting of a pair of opposite magnetic charges with the field $\vec{B}_{m G}=$ $-\vec{\nabla}\left(\vec{m}_{G} \cdot \vec{r} / r^{3}\right)$ related to the gradient of scalar potential, is taken to be at the origin. The field at the dipole (ie, at the origin) due to the magnetic charge $p$ at location $\vec{r}_{p}$ is $\vec{B}_{p}=-p \vec{r}_{p} / r_{p}^{3}$ with $\vec{\nabla} \cdot \vec{B}_{p}=4 \pi p \delta^{3}\left(\vec{r}-\vec{r}_{p}\right)$. The interaction energy of these fields is

$$
\begin{aligned}
& U_{\mathrm{int}}=\frac{1}{4 \pi} \int \vec{B}_{m G} \cdot \vec{B}_{p} \mathrm{~d} v=-\frac{1}{4 \pi} \int \vec{\nabla}\left(\frac{\vec{m}_{G} \cdot \vec{r}}{r^{3}}\right) \cdot \vec{B}_{p} \mathrm{~d} v, \\
& U_{\mathrm{int}}=-\frac{1}{4 \pi} \int\left[\vec{\nabla} \cdot\left(\frac{\vec{m}_{G} \cdot \vec{r}}{r^{3}} \vec{B}_{p}\right)-\frac{\vec{m}_{G} \cdot \vec{r}}{r^{3}} \vec{\nabla} \cdot \vec{B}_{p}\right] \mathrm{d} v .
\end{aligned}
$$

Using the divergence theorem, the expression can be transformed to

$$
U_{\text {int }}=-\frac{1}{4 \pi}\left(\int_{A \text { at } \infty} \frac{\vec{m}_{G} \cdot \vec{r}}{r^{3}} \vec{B}_{p} \cdot \mathrm{d} \vec{A}-\int \frac{\vec{m}_{G} \cdot \vec{r}}{r^{3}} \vec{\nabla} \cdot \vec{B}_{p} \mathrm{~d} v\right) .
$$

$\vec{B}_{p} \propto 1 / r^{2}, \mathrm{~d} \vec{A} \propto r^{2} ;$ therefore, the first integral vanishes with $1 / r$ as $r$ becomes very large. And if we use the definition $\vec{\nabla} \cdot \vec{B}_{p}=4 \pi p \delta^{3}\left(\vec{r}-\vec{r}_{p}\right)$, we obtain

$$
U_{\mathrm{int}}=-\vec{m}_{G} \cdot \vec{B}_{p}
$$

As a result of these calculations, the interaction of a Gilbertian magnetic charge with a Gilbertian magnetic dipole does conserve energy.

\subsection{Gilbertian magnetic charge with an associated \\ Dirac string and Gilbertian magnetic dipole}

The magnetic field of Gilbertian magnetic charge $p$ at the origin with an associated Dirac string can be defined as $\vec{B}=\vec{B}_{p}+\vec{B}_{s}$. The interaction energy of a Gilbertian magnetic charge with a Gilbertian magnetic dipole $\vec{m}_{G}$ can be obtained using the formulation in sections 2.2 and 2.3

$$
U_{\mathrm{int}}=\frac{1}{4 \pi} \int \vec{B}_{m G} \cdot\left(\vec{B}_{p}+\vec{B}_{s}\right) \mathrm{d} v=-2 \vec{m}_{G} \cdot \vec{B}_{p}
$$

The implication is that the interaction energy of a Gilbertian magnetic charge with an associated Dirac string and a Gilbertian magnetic dipole is not conserved. 
2.5 Gilbertian magnetic charge and Amperian magnetic dipole

Consider the interaction energy of a Gilbertian magnetic charge $p$ at the origin with an Amperian magnetic dipole $\left(\vec{B}_{m A}=[3(\vec{m} \cdot \hat{r}) \hat{r}-\vec{m}] / r^{3}\right.$ with $\left.\vec{\nabla} \cdot \vec{B}_{m A}=0\right)$,

$$
\begin{gathered}
U_{\mathrm{int}}=\frac{1}{4 \pi} \int \vec{B}_{m A} \cdot \vec{B}_{p} \mathrm{~d} v=-\frac{1}{4 \pi} \int \vec{B}_{m A} \cdot \vec{\nabla} \frac{p}{r} \mathrm{~d} v, \\
U_{\mathrm{int}}=-\frac{1}{4 \pi} \int\left[\vec{\nabla} \cdot\left(\frac{p}{r} \vec{B}_{m A}\right)-\frac{p}{r} \vec{\nabla} \cdot \vec{B}_{m A}\right] \mathrm{d} v .
\end{gathered}
$$

If we use the definition $\vec{\nabla} \cdot \vec{B}_{m A}=0$, we obtain

$$
U_{\text {int }}=-\frac{1}{4 \pi} \int \vec{\nabla} \cdot\left(\frac{p}{r} \vec{B}_{m A}\right) \mathrm{d} v=-\frac{1}{4 \pi} \int_{A \text { at } \infty} \frac{p}{r} \vec{B}_{m A} \cdot \mathrm{d} \vec{A}
$$

$\vec{B}_{m A} \propto 1 / r^{3}, \mathrm{~d} \vec{A} \propto r^{2}$; therefore, the integrand vanishes with $1 / r$ as $r$ becomes very large. Thus, we obtain

$$
U_{\text {int }}=0 \text {. }
$$

As a result of these calculations, the interaction of a Gilbertian magnetic charge with a "point" Amperian magnetic dipole does not conserve energy.

\subsection{Gilbertian magnetic charge with an associated \\ Dirac string and Amperian magnetic dipole}

Consider the interaction energy between a Gilbertian magnetic charge $p$ at the origin with an associated Dirac string and Amperian magnetic dipole with the field $\vec{B}_{m A}$,

$$
\begin{gathered}
U_{\mathrm{int}}=\frac{1}{4 \pi} \int \vec{B}_{m A} \cdot\left(\vec{B}_{p}+\vec{B}_{s}\right) \mathrm{d} v \\
U_{\mathrm{int}}=-\frac{1}{4 \pi} \int\left(\vec{\nabla} \cdot \frac{p \vec{B}_{m A}}{r}-\frac{p \vec{\nabla} \cdot \vec{B}_{m A}}{r}-\vec{B}_{m A} \cdot \vec{B}_{s}\right) \mathrm{d} v .
\end{gathered}
$$

If we use the divergence theorem and the definition $\vec{\nabla}$. $\vec{B}_{m A}=0$, the integral can be transformed to

$$
U_{\mathrm{int}}=-\frac{1}{4 \pi} \int_{A \text { at } \infty} \frac{p}{r} \vec{B}_{m A} \cdot \mathrm{d} \vec{A}+\frac{1}{4 \pi} \int \vec{B}_{m A} \cdot \vec{B}_{s} \mathrm{~d} v
$$

$\vec{B}_{m A} \propto 1 / r^{3}, \mathrm{~d} \vec{A} \propto r^{2}$; therefore, the first integral vanishes with $1 / r$ as $r$ becomes very large. Thus, we obtain

$$
\begin{aligned}
U_{\text {int }} & =\frac{1}{4 \pi} \int_{\text {int }} \vec{B}_{m A} \cdot \vec{B}_{s} \mathrm{~d} v=p \int \vec{B}_{m A} \cdot \vec{\delta}_{s} \mathrm{~d} v \\
& =p \int_{\hat{n}_{A} \| \hat{s}} \int_{\hat{n}_{A} \| \hat{s}}\left(\vec{B}_{m A} \cdot \vec{\delta}_{m A}\right)(\mathrm{d} \vec{s} \cdot \mathrm{d} \vec{A}) \\
& \left.=p \int_{s} \vec{B}_{m A} \cdot \mathrm{d} \vec{s}=-p \vec{\delta}_{s} \cdot \mathrm{d} A\right) \\
& =p \varphi_{m A}(0)=-p \frac{\vec{m}_{A} \cdot\left(\vec{r}-\vec{r}_{m}\right)}{\left|\vec{r}-\vec{r}_{m}\right|^{3}} \cdot \mathrm{d} \vec{s} \\
r_{m}^{3} & =-\vec{m}_{A} \cdot \vec{B}_{p} .
\end{aligned}
$$

The implication is that the interaction energy between a Gilbertian magnetic charge with an associated Dirac string and a "point" Amperian magnetic dipole does conserve energy.

\section{Conclusion}

We proposed that a Dirac string is defined for only one magnetic monopole in the medium. If there is another magnetic monopole in calculable distance to the resting monopole in the medium, we cannot introduce the definition of a Dirac string. The proposition that there could exist at most one Dirac monopole/string in the universe is probably not what Dirac had in mind, although it is a logical possibility.

\section{Acknowledgements}

Thanks to Kirk T. McDonald for his guidance and support. This work was supported by the Scientific and Technological Research Council of Turkey (Tübitak), Grant No 117E508.

\section{REFERENCES}

[1] P. A. M. Dirac, "Quantised Singularities in the Electromagnetic Field", Proc. Roy. Soc. London vol. A133, pp. 60-72, 1931.

[2] P. A. M. Dirac, "The Theory of Magnetic Monopoles", Phys. Rev. vol. 74, pp. 817-830, 1948.

[3] J. Schwinger, "Magnetic Charge and Quantum Field Theory", Phys. Rev. vol. 144, pp. 1087-1093, 1966.

[4] D. Zwanziger, "Local-Lagrangian Quantum Field Theory of Electric and Magnetic Charges", Phys. Rev. D, vol. 3, pp. 880-891, 1971.

[5] T. T. Wu and C. N. Yang, "Concept of Nonintegrable Phase Factors and Global Formulation of Gauge Fields", Phys. Rev. D, vol. 12, pp. 3845-3857, 1975.

[6] H. J. Lipkin and M. Peshkin, "Magnetic Monopoles, Electric Currents, and Dirac Strings",Phys. Lett. B, vol. 179, pp. 109-114, 1986.

[7] H. J. Lipkin and M. Peshkin, "Magnetic Monopoles and Dipoles in Quantum Mechanics", Ann. N. Y. Acad. Sci. vol. 480, pp. 210-216, 1986.

[8] E. Comay, "Interactions of Static Electric and Magnetic Fields", Lett. Nuovo Cim. vol. 38, pp. 421-422, 1983.

[9] E. Comay, "A Difference Between Solenoid and Magnetic Spin", J. Mag. Mag. Mat. vol. 43, pp. 59-60, 1984.

[10] E. Comay, "Geometry and Charge-Monopole Systems", Phys. Lett. B, vol. 187, pp. 111-114, 1987.

[11] J. M. Getino, O. Rojo and H. Rubio, "Interaction between Electric Currents and Magnetic Monopoles", Europhys. Lett. vol. 15, pp. 821-825, 1991.

[12] K. T. McDonald, "Comay's Paradox: Do Magnetic Charges Conserve Energy?", http://www.physics.princeton.edu/ modonald/examples/comay.pdf, July 16, 2015.

Received 22 March 2019 